\title{
Noninvasive markers: a double-edged sword that strati- fies nonalcoholic steatohepatitis
}

\author{
Goh Eun Chung and Donghee Kim \\ Department of Internal Medicine, Healthcare Research Institute, Gangnam Healthcare Center, Seoul National University Hospital, \\ Seoul, Korea
}

Keywords: Fatty liver; Cytokeratin 18; Apoptosis; Steatohepatitis; Fibrosis

\section{See Article on Page 120}

Nonalcoholic fatty liver disease (NAFLD) has been recognized as the most common liver disease with an estimated prevalence of $20-30 \%$ in the Western world and $16-33 \%$ in Korea. ${ }^{1-3}$ NAFLD is considered to be the hepatic manifestation of metabolic syndrome, because the mechanism underlying the development of NAFLD has been linked to insulin resistance and metabolic syndrome. ${ }^{4-6}$ Therefore, NAFLD is closely related to obesity, dyslipidemia, type II diabetes and coronary artery disease. ${ }^{7-9}$

NAFLD encompasses a broad spectrum of hepatic dysfunction ranging from nonalcoholic fatty liver (NAFL) to nonalcoholic steatohepatitis (NASH), cirrhosis, and hepatocellular carcinoma. ${ }^{10,11}$ Although NAFL typically follows a benign clinical condition, NASH is a potentially progressive disease given that $25 \%$ of patients may develop cirrhosis, complications of portal hypertension, and hepatocellular carcinoma. ${ }^{12,13}$ Furthermore, patients with NASH have increased mortality compared to the general population. ${ }^{14,15}$ A recent large population study shows that NAFL was not associated with higher mortality risk after a median follow-up of 15 years. $^{16}$ Further analysis shows that NAFLD patients without advanced fibrosis do not have a higher mortality risk and mortality increases as fibrosis advances. ${ }^{16}$ Therefore, distinguishing between NAFL and NASH with or without fibrosis is clinically important for prognosis and therapeutic interventions.

Although there is past and ongoing research for various noninvasive tests to distinguish NASH from NAFL, liver biopsy remains the reference standard method to diagnose the presence of NASH and assess the severity of liver injury. However, liver biopsies are not easily performed because of several limitations. First, it is an invasive procedure with potentially significant complications occurring with a morbidity and mortality rate of $3 \%$ and $0.03 \%$, respectively. ${ }^{17,18}$ Second, a percutaneous liver biopsy captures an extremely tiny portion of the liver $(\sim 1 / 50,000$ of total mass of the liver), leading to significant sampling variability $(25 \%-40 \%){ }^{19,20}$ In addition, the extent of variation from observer interpretation by expert pathologists may be as high as $20 \%{ }^{21}$

Therefore, there is an urgent need to investigate and validate a reproducible noninvasive test that distinguishes NASH from NAFL. Several studies have tried to identify potential noninvasive biomarkers, which are markers of the key pathways believed to be associated with NASH pathogenesis. These pathways include oxidative stress, inflammation, insulin resistance, and apoptosis. ${ }^{22,23}$

\section{Abbreviations:}

AUROC, area under receiver operating characteristic curve; CK-18, Cytokeratin 18; Cl, confidence interval; NAFL, non-alcoholic fatty liver; NAFLD, non-alcoholic fatty liver disease; NASH, non-alcoholic steatohepatitis

\section{Corresponding author : Donghee Kim}

Department of Internal Medicine, Healthcare Research Institute, Healthcare System Gangnam Center, Seoul National University Hospital, 39FL., Gangnam Finance Center, 737 Yeoksam-dong, Gangnam-gu, Seoul 135-984, Korea

Tel. +82-2-2112-5574, Fax. +82-2-2112-5635

E-mail: messmd@chol.com

Received : May 10, 2013/ Revised : May 15, 2013 
Goh Eun Chung et al. Noninvasive markers: a double-edged sword that stratifies nonalcoholic steatohepatitis

High-sensitivity C-reactive protein, plasma pentraxin 3, tumor necrosis factor-alpha, and interleukin- 6 were studied, but current results were not sufficiently accurate for clinical use. ${ }^{24-28}$ NashTest (Biopredictive Paris, France), which uses a proprietary algorithm, has the area under the receiver operating characteristic curve (AUROC) of $0.79 .^{29}$ However, this index has only been validated in the original studies and further validation is needed for clinical implication.

Hepatic apoptosis is suggested to play a critical role in liver injury and progression of NAFLD. ${ }^{30}$ Cytokeratin 18 (CK-18) is the major intermediate filament protein in the liver and linked to the morphological changes of apoptosis. In the original study, CK-18 fragments were tested in the livers and plasma for biopsy-proven NAFLD and controls, and caspase-3 generated CK-18 activation in the blood that was an independent predictor of NASH. ${ }^{31}$ Subsequently, a multicenter validation study was performed and included 139 patients with biopsy-proven NAFLD from the NASH Clinical Research Network and 150 age-matched healthy controls. ${ }^{32}$ The AUROC for NASH was estimated to be 0.83 (95\% Confidence Interval (CI) 0.75-0.91). ${ }^{32}$ CK-18 levels were an independent predictor of both NASH and severity of NAFLD in this study. ${ }^{32}$ CK-18 is the only marker for NASH that has been externally validated in nine studies, enrolling a total of 856 NAFLD patients of various ethnicities, obesity, and diabetes status in a recent meta-analysis. $^{33}$ AUROC, sensitivity, specificity are 0.82 (95\% Cl 0.76-0.88), $0.78(0.65-0.91)$, and $0.86(0.75-0.97)$, respectively. ${ }^{33}$ In addition, CK-18 was found to be the most accurate biomarker for the diagnosis of NAFLD and NASH in combination with fibroblast growth factor $21 .^{34}$

In this study by Kim et al, they conducted a prospective cohort study with 108 patients with biopsy-proven NAFLD from 10 participant centers across Korea to i) identify the useful clinical parameters in a noninvasive approach to distinguish NASH from NAFL and ii) to determine whether these levels would be related to the severity of liver injury in patients with NASH. ${ }^{35} \mathrm{CK}-18$ levels had a positive correlation with NAFLD activity score and subtype. The serum CK-18 level was significantly higher in the NAFLD subtype 3 or 4 group (NASH group) than the NAFLD subtype 1 or 2 (NAFL group). A CK-18 cutoff value of $235.5 \mathrm{U} / \mathrm{L}$ showed a sensitivity of $69.9 \%$, a specificity of $64.9 \%$, and positive and negative predictive value of $75.5 \%$ and $57.1 \%$, respectively, for the diagnosis of NASH. The strength of this multicenter prospective study is that serum CK-18 has potential as a non-invasive biochemical marker to distinguish NASH from NAFL in Korean patients for the first time. In addition, this study provided valuable information that the prevalence of significant fibrosis was $35.2 \%$ (38/108) in adults with a mean age of 39 . We definitely considered that more patients with more severe disease were included in the study than NAFLD patients from the general population due to the enrollment of liver biopsy-proven NAFLD patients in the tertiary hospital. Currently, to distinguish between NAFL and NASH with or without fibrosis, we needed to use noninvasive markers of fibrosis, which are useful in monitoring, counseling, and in some cases, making treatment decisions in patients with NAFLD in Korea and in the Western world.

In this study, the AUROC of CK-18 was relatively low (0.605) compared to previous studies with a relatively high AUROC up to 0.83 for the diagnosis of NASH. ${ }^{32,33}$ An NAFLD activity score consists of the weighted sum of each of the following: steatosis, lobular inflammation, and the presence of hepatocyte ballooning. The major difference between the NAFLD activity score, used as definition in previous studies, and the NAFLD subtype category, used as definition of NASH in this study, is that the NAFLD subtype incorporated fibrosis into its definition. ${ }^{36}$ Generally, the original criteria for the NAFLD subtype used in this study does not use commonly compared criteria according to the consensus of the NASH Clinical Research Network Pathology Committee used in other studies. ${ }^{30,32}$ Therefore, it is important to carefully consider the differences and similarities among several histologic scoring systems when assessing disease severity, the accuracy of noninvasive markers, and the prognosis of NAFLD. ${ }^{36,37}$

There were noteworthy limitations in this study. To confirm whether CK-18 levels are an independent biomarker of NASH in this study, the statistical significance of CK-18 using multivariate logistic regression analysis might be helpful in drawing a more evident conclusion. In addition, as the author stated, the patients were recruited in a tertiary academic hospital where all the patients had a liver biopsy. Hence, patients with more severe disease activity than NAFLD patients from the general population were included in this cohort, constituting a selection bias.

In conclusion, this study validated serum CK-18 as a biochemical marker to distinguish NASH from NAFL in Korean patients. Because this article has multi-institutional data, it has clinical value for Korean NAFLD patients. To confirm these results, larger validation studies and longitudinal prospective analyses are required in the future.

\section{Conflicts of Interest}

The authors have no conflicts to disclose. 


\section{REFERENCES}

1. Vernon G, Baranova A, Younossi ZM. Systematic review: the epidemiology and natural history of non-alcoholic fatty liver disease and non-alcoholic steatohepatitis in adults. Aliment Pharmacol Ther 2011;34:274-285.

2. Choi SY, Kim D, Kim HJ, Kang JH, Chung SJ, Park MJ, et al. The relation between non-alcoholic fatty liver disease and the risk of coronary heart disease in Koreans. Am J Gastroenterol 2009;104:19531960.

3. Bae JC, Cho YK, Lee WY, Seo HI, Rhee EJ, Park SE, et al. Impact of nonalcoholic fatty liver disease on insulin resistance in relation to HbA1c levels in nondiabetic subjects. Am J Gastroenterol 2010;105:2389-2395.

4. Almeda-Valdes P, Cuevas-Ramos D, Aguilar-Salinas CA. Metabolic syndrome and non-alcoholic fatty liver disease. Ann Hepatol 2009;8(Suppl 1):S18-S24.

5. Chen SH, He F, Zhou HL, Wu HR, Xia C, Li YM. Relationship between nonalcoholic fatty liver disease and metabolic syndrome. J Dig Dis 2011;12:125-130.

6. Khashab MA, Liangpunsakul S, Chalasani N. Nonalcoholic fatty liver disease as a component of the metabolic syndrome. Curr Gastroenterol Rep 2008;10:73-80.

7. Choi SY, Kim D, Kang JH, Park MJ, Kim YS, Lim SH, et al. Nonalcoholic fatty liver disease as a risk factor of cardiovascular disease: relation of non-alcoholic fatty liver disease to carotid atherosclerosis. Korean J Hepatol 2008;14:77-88.

8. Targher G, Marra F, Marchesini G. Increased risk of cardiovascular disease in non-alcoholic fatty liver disease: causal effect or epiphenomenon? Diabetologia 2008;51:1947-1953.

9. Kim D, Choi SY, Park EH, Lee W, Kang JH, Kim W, et al. Nonalcoholic fatty liver disease is associated with coronary artery calcification. Hepatology 2012;56:605-613.

10. Clark JM. The epidemiology of nonalcoholic fatty liver disease in adults. J Clin Gastroenterol 2006;40(Suppl 1):S5-S10.

11. Erickson SK. Nonalcoholic fatty liver disease. J Lipid Res 2009;50(Suppl):S412-S416.

12. Adams LA, Lymp JF, St Sauver J, Sanderson SO, Lindor KD, Feldstein $A$, et al. The natural history of nonalcoholic fatty liver disease: a population-based cohort study. Gastroenterology 2005;129:113121.

13. Ekstedt M, Franzen LE, Mathiesen UL, Thorelius L, Holmqvist M, Bodemar $\mathrm{G}$, et al. Long-term follow-up of patients with NAFLD and elevated liver enzymes. Hepatology 2006;44:865-873.

14. Rafiq N, Bai C, Fang Y, Srishord M, McCullough A, Gramlich T, et al. Long-term follow-up of patients with nonalcoholic fatty liver. Clin Gastroenterol Hepatol 2009;7:234-238.

15. Soderberg C, Stal P, Askling J, Glaumann H, Lindberg G, Marmur
J, et al. Decreased survival of subjects with elevated liver function tests during a 28-year follow-up. Hepatology 2010;51:595-602.

16. Kim D, Kim WR, Kim HJ, Therneau TM. Association between noninvasive fibrosis markers and mortality among adults with nonalcoholic fatty liver disease in the United States. Hepatology 2013:57:1357-1365.

17. Wieckowska A, Feldstein AE. Diagnosis of nonalcoholic fatty liver disease: invasive versus noninvasive. Semin Liver Dis 2008;28:386395.

18. Piccinino F, Sagnelli E, Pasquale G, Giusti G. Complications following percutaneous liver biopsy. A multicentre retrospective study on 68,276 biopsies. J Hepatol 1986;2:165-173.

19. Ratziu V, Charlotte F, Heurtier A, Gombert S, Giral P, Bruckert E, et al. Sampling variability of liver biopsy in nonalcoholic fatty liver disease. Gastroenterology 2005;128:1898-1906.

20. Merriman RB, Ferrell LD, Patti MG, Weston SR, Pabst MS, Aouizerat $\mathrm{BE}$, et al. Correlation of paired liver biopsies in morbidly obese patients with suspected nonalcoholic fatty liver disease. Hepatology 2006;44:874-880.

21. Bravo AA, Sheth SG, Chopra S. Liver biopsy. N Engl J Med 2001;344:495-500.

22. Oh MK, Winn J, Poordad F. Review article: diagnosis and treatment of non-alcoholic fatty liver disease. Aliment Pharmacol Ther 2008;28:503-522.

23. Dowman JK, Tomlinson JW, Newsome PN. Systematic review: the diagnosis and staging of non-alcoholic fatty liver disease and nonalcoholic steatohepatitis. Aliment Pharmacol Ther 2011;33:525540.

24. Campos GM, Bambha K, Vittinghoff E, Rabl C, Posselt AM, Ciovica $R$, et al. A clinical scoring system for predicting nonalcoholic steatohepatitis in morbidly obese patients. Hepatology 2008;47:19161923.

25. Manco M, Marcellini M, Giannone G, Nobili V. Correlation of serum TNF-alpha levels and histologic liver injury scores in pediatric nonalcoholic fatty liver disease. Am J Clin Pathol 2007;127:954-960.

26. Solga SF, Alkhuraishe A, Cope K, Tabesh A, Clark JM, Torbenson $M$, et al. Breath biomarkers and non-alcoholic fatty liver disease: preliminary observations. Biomarkers 2006;11:174-183.

27. Sookoian S, Castano G, Burgueno AL, Gianotti TF, Rosselli MS, Pirola CJ. A diagnostic model to differentiate simple steatosis from nonalcoholic steatohepatitis based on the likelihood ratio form of Bayes theorem. Clin Biochem 2009;42:624-629.

28. Barsic N, Lerotic I, Smircic-Dunnjak L, Tomasic V, Duvnjak M. Overview and developments in noninvasive diagnosis of nonalcoholic fatty liver disease. World J Gastroenterol 2012;18:3945-3954.

29. Poynard T, Ratziu V, Charlotte F, Messous D, Munteanu M, ImbertBismut $F$, et al. Diagnostic value of biochemical markers (NashTest) for the prediction of non alcoholo steato hepatitis in patients with 
non-alcoholic fatty liver disease. BMC Gastroenterol 2006;6:34.

30. Feldstein AE, Canbay A, Angulo P, Taniai M, Burgart LJ, Lindor $K D$, et al. Hepatocyte apoptosis and fas expression are prominent features of human nonalcoholic steatohepatitis. Gastroenterology 2003; 125:437-443.

31. Wieckowska A, Zein NN, Yerian LM, Lopez AR, McCullough AJ, Feldstein AE. In vivo assessment of liver cell apoptosis as a novel biomarker of disease severity in nonalcoholic fatty liver disease. Hepatology 2006;44:27-33.

32. Feldstein AE, Wieckowska A, Lopez AR, Liu YC, Zein NN, MCCullough AJ. Cytokeratin-18 fragment levels as noninvasive biomarkers for nonalcoholic steatohepatitis: a multicenter validation study. Hepatology 2009;50:1072-1078.

33. Musso G, Gambino R, Cassader M, Pagano G. Meta-analysis: natural history of non-alcoholic fatty liver disease (NAFLD) and diagnos- tic accuracy of non-invasive tests for liver disease severity. Ann Med 2011;43:617-649.

34. Shen J, Chan HL, Wong GL, Choi PC, Chan AW, Chan HY, et al. Non-invasive diagnosis of non-alcoholic steatohepatitis by combined serum biomarkers. J Hepatol 2012;56:1363-1370.

35. Kim YS, Jung ES, Yoon SK, Bae SH, Choi JY, Song MJ, et al. Noninvasive predictors of nonalcoholic steathepatitis in Korean patients with histologically proven nonalcoholic fatty liver disease. Clin Mol Hepatol 2013;19:120-130.

36. Pagadala MR, McCullough AJ. The relevance of liver histology to predicting clinically meaningful outcomes in nonalcoholic steatohepatitis. Clin Liver Dis 2012;16:487-504.

37. Adams LA, Feldstein AE. Non-invasive diagnosis of nonalcoholic fatty liver and nonalcoholic steatohepatitis. J Dig Dis 2011;12:10-16. 